\title{
Éclosion d'hépatite A dans la collectivité, qui touche de manière disproportionnée les hommes ayant des relations sexuelles avec des hommes à Toronto, Canada, janvier 2017-novembre 2018
}

\author{
H Sachdeva ${ }^{1,2 *}$, M Benusic ${ }^{3}$, S Ota ${ }^{1}$, R Stuart ${ }^{1}$, J Maclachlan ${ }^{1}$, V Dubey ${ }^{1,2}$, A Andonov ${ }^{4}$
}

\section{Résumé}

Contexte : Fin 2016 et début 2017, un certain nombre de pays ont commencé à signaler des éclosions de virus de l'hépatite $\mathrm{A}(\mathrm{VHA})$ impliquant une transmission de personne à personne entre hommes ayant des relations sexuelles avec des hommes (HARSAH), consommateurs de drogues illicites et personnes sans abri ou mal-logées.

Objectif : Décrire l'épidémiologie et les interventions de santé publique face à une éclosion de VHA qui touche de manière disproportionnée les HARSAH à Toronto, au Canada, de janvier 2017 à novembre 2018.

Méthodologie : À la suite de l'augmentation du nombre de cas de VHA chez les HARSAH signalés dans d'autres pays, tous les cas de VHA non liés aux voyages et signalés du $1^{\text {er }}$ juin 2017 au $1^{\text {er }}$ novembre 2018 ont fait l'objet d'une surveillance accrue, y compris une analyse rétrospective des cas signalés entre janvier 2017 et juin 2017. Une analyse descriptive et un séquençage viral ont été effectués pour décrire les modes de transmission de personne à personne et les interventions ciblées. Les stratégies de contrôle comprenaient des interventions visant à promouvoir le recours à la vaccination contre le VHA avant l'exposition, y compris des campagnes sur les réseaux sociaux destinées aux HARSAH, ainsi que des messages destinés aux professionnels de la santé et aux cliniques de vaccination.

Résultats : D'après les définitions de cas d'éclosion, 52 cas confirmés et probables de VHA ont été identifiés. Plus de $80 \%$ des cas d'éclosion concernaient des hommes $(n=43 / 52)$ et, parmi ceux pour lesquels des données étaient disponibles, $64 \%(n=25 / 39)$ ont déclaré une exposition à des HARSAH. Les données sur l'hospitalisation étaient disponibles pour 51 cas; $56 \%$ des cas confirmés $(n=23 / 41)$ et $40 \%$ des cas probables $(n=4 / 10)$ ont nécessité une hospitalisation. Parmi les cas avec des échantillons de sérum comportant un séquençage du VHA, $83 \%(n=30 / 36)$ présentaient l'une des trois souches prévalentes dans les éclosions chez les HARSAH mondialement; $72 \%(n=26 / 36)$ étaient du type VRD_521_2016, qui avait été détecté dans des éclosions européennes récemment signalées chez les HARSAH. Une campagne de promotion ciblée de la vaccination, financée par des fonds publics sur des plateformes de réseaux sociaux populaires auprès des HARSAH et des cliniques de vaccination ciblées, a été mise sur pied pour promouvoir la sensibilisation au VHA et l'adoption du vaccin chez les HARSAH.

Conclusion : Des éclosions de VHA, attribuées à la transmission de personne à personne de souches de VHA touchant de façon disproportionnée les HARSAH et susceptibles d'avoir été importées d'éclosions touchant les HARSAH à l'échelle mondiale se sont maintenant produites au Canada. Le séquençage génétique du VHA, l'analyse des facteurs de risque des cas, le suivi des tendances de la couverture vaccinale dans les groupes à haut risque et le lancement de campagnes de vaccination visant à éliminer les obstacles à la couverture vaccinale avant exposition au VHA chez les HARSAH pourraient prévenir de futures éclosions.
Cette oeuvre est mise à la disposition selon les termes de la licence internationale Creative Commons Attribution 4.0

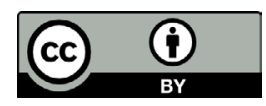

Affiliations

${ }^{1}$ Contrôle des maladies transmissibles, Bureau de santé publique de Toronto, Toronto, ON

2 École de santé publique Dalla Lana, Université de Toronto, Toronto, ON

${ }^{3}$ Programme de résidence en santé publique et en médecine préventive, Université de Toronto, Toronto, ON

${ }^{4}$ Laboratoire national de microbiologie, Winnipeg, MB

*Correspondance :

herveen.sachdeva@toronto.ca
Citation proposée : Sachdeva H, Benusic M, Ota S, Stuart R, Maclachlan J, Dubey V, Andonov A. Éclosion

d'hépatite $A$ dans la collectivité, qui touche de manière disproportionnée les hommes ayant des relations sexuelles avec des hommes à Toronto, Canada, janvier 2017-novembre 2018. Relevé des maladies transmissibles au Canada 2019;45(10):289-95. https://doi.org/10.14745/ccdr.v45i10a03f

Mots-clés : VHA, éclosion, hommes ayant des relations sexuelles avec des hommes, promotion du vaccin, HARSAH 


\section{Introduction}

L'infection par le virus de l'hépatite $A(V H A)$ est endémique dans les pays en développement et constitue l'une des maladies les plus courantes évitables par la vaccination chez les voyageurs (1). La maladie clinique se manifeste après une période d'incubation de 15 à 50 jours et commence généralement par l'apparition soudaine de fièvre, de nausées et de douleurs abdominales, suivies d'un ictère (2). Bien que la plupart des cas guérissent sans traitement, $25 \%$ des cas d'adultes nécessitent une hospitalisation (1). Un taux de létalité de 2,2\% a été observé chez les personnes âgées de plus de 60 ans (3).

La principale source de VHA est la nourriture ou l'eau contaminée, mais la transmission de personne à personne a également été signalée, en particulier par contact sexuel anal-oral ou digital-anal. Les hommes ayant des relations sexuelles avec des hommes (HARSAH) ont été identifiés comme un groupe à risque plus élevé de transmission du VHA de personne à personne (4).

Fin 2016 et début 2017, une série d'éclosions de VHA a été signalée dans des pays où les taux de VHA étaient auparavant faibles. Plus précisément, les États-Unis, 15 pays de la région européenne et le Chili ont fait état d'une augmentation de I'incidence chez les HARSAH (5). En Europe, trois souches du génotype 1A en co-circulation (VRD_521_2016, V16-25801 et RIVM-HAV16-090) ont été signalées, leurs origines étant liées à l'Amérique centrale et à l'Asie (4). De plus, au cours de cette période, des éclosions avec des souches virales de génotype $1 \mathrm{~b}$ ont été signalées aux États-Unis chez des personnes ayant déclaré avoir consommé des drogues illicites ou être des sansabri (6).

En Ontario, l'administration de deux doses du vaccin contre le VHA est financée par la province pour trois groupes : les HARSAH, ceux qui utilisent des drogues intraveineuses et ceux qui ont une maladie hépatique préexistante (7). En Ontario, la confirmation d'un cas de VHA nécessite une sérologie avec des symptômes compatibles ou un lien épidémiologique avec un cas confirmé (8). Le Bureau de santé publique de Toronto reçoit les rapports de cas suspects et confirmés de VHA, puis les examine et en fait le suivi. De 2012 à 2016, l'incidence moyenne de VHA déclarés était de 32 cas par année (9).

Compte tenu des différentes populations à risque élevé qui sont touchées par la transmission du VHA de personne à personne à l'échelle mondiale $(5,6)$, en juin 2017, le Bureau de santé publique de Toronto a instauré une surveillance accrue des cas de VHA, demandé le génotypage de l'hépatite $A$ et le séquençage viral de tous les nouveaux cas déclarés, et effectué une étude rétrospective des facteurs de risque des cas signalés à Toronto depuis janvier 2017. L'analyse initiale effectuée en août
2017 a montré que le nombre de cas correspondait à la moyenne des cinq années précédentes pour cette période. Toutefois, près de $50 \%$ des cas n'ont pas signalé de voyage pendant la période d'incubation, par rapport à une moyenne de $30 \%$ pour les cinq années précédentes. En outre, près de $40 \%$ étaient des HARSAH, par rapport à la moyenne quinquennale précédente de $4 \%$. Les cas de VHA contractés localement chez les HARSAH ont continué d'être détectés en septembre 2017 et une éclosion a été officiellement déclarée en octobre 2017.

L'objectif de ce rapport est de décrire l'épidémiologie de la transmission du VHA contracté de personne à personne localement à Toronto et les mesures en santé publique pour gérer une éclosion de VHA touchant de façon disproportionnée les HARSAH de janvier 2017 à novembre 2018.

\section{Méthodologie}

\section{Détection d'éclosion et enquête}

Aux fins de la présente enquête, une définition de cas d'éclosion a été établie pour inclure tous les cas contractés localement. Les cas d'éclosion confirmés étaient définis comme des résidents ou des visiteurs de Toronto :

- Qui répondaient à la définition provinciale de cas pour un cas confirmé de VHA (8)

- Dont la date de déclaration était le 1er janvier 2017 ou après cette date

- Qui n'avaient pas voyagé au cours de leur période d'infection

- Qui n'avaient aucun lien épidémiologique avec un cas lié à un voyage

Les cas probables ont été définis comme des patients dont la date de déclaration était le $1^{\mathrm{er}}$ janvier 2017 ou après cette date et qui :

- Répondaient à la définition provinciale de cas pour un cas confirmé de VHA

- Avaient voyagé pendant la période d'infection ou avaient un lien épidémiologique avec un voyageur

- Avaient un facteur de risque de transmission du VHA de personne à personne (sans-abri ou mal-logés, consommation de drogues illicites ou HARSAH)

\section{Gestion des cas et des personnes d'intérêt}

La gestion de cas a été amorcée au moyen d'une entrevue téléphonique guidée par un questionnaire normalisé de Santé publique de l'Ontario (10). Conformément aux procédures habituelles de gestion de cas, on a eu recours aux professionnels de la santé, au personnel des refuges et au personnel spécialisé dans la réduction des méfaits pour trouver les personnes difficiles à joindre. Des conseils ont été fournis pour réduire le risque de transmission à d'autres personnes. Des personnes 
d'intérêt ont été interviewées pour déterminer leur admissibilité à la prophylaxie post-exposition. Les données sur les cas et les personnes d'intérêt ont été saisies dans le Système d'information en santé publique intégré (SISPi) de l'Ontario.

\section{Séquençage moléculaire}

Le génotypage et le séquençage moléculaire du virus de I'hépatite A ne sont pas systématiquement effectués en Ontario, mais ont été faits dans le cadre de cette enquête sur l'éclosion. Les échantillons de sérum envoyés aux laboratoires de Santé publique de l'Ontario ont été envoyés au Laboratoire national de microbiologie à Winnipeg, au Manitoba, pour le génotypage et le séquençage moléculaire, dans la mesure du possible, selon la disponibilité d'un échantillon sérologique au laboratoire de diagnostic. Les résultats du séquençage ont été comparés aux souches provenant d'éclosions à l'échelle mondiale.

\section{Analyse}

Une liste de cas d'éclosion de VHA du $1^{\text {er }}$ janvier 2017 au 30 novembre 2018 a été extraite du Système d'information en santé publique intégré (SISPi), comprenant des données sur l'âge, le sexe, l'adresse, l'épisode et la date déclarée, les facteurs de risque et les résultats de laboratoire.

L'analyse statistique a été réalisée avec Stata Statistical Software : Version 15 (College Station, Texas, États-Unis : StataCorp LLC). Les rapports de cotes ont été analysés par régression logistique simple. La signification statistique a été fixée à alpha moins de 0,05.

Les adresses des éclosions confirmées ont été tracées à l'aide d'ArcMap 10.5 (Redlands, Californie, États-Unis : Environmental Systems Research Institute). Une carte thermique a été produite en utilisant la densité en un point, et l'indice global de Moran (test d'autocorrélation spatiale) a été appliqué pour évaluer le caractère aléatoire de la distribution spatiale après avoir appliqué une grille cartésienne pour l'agrégation des points.

À Toronto, certains vaccins financés par la province, y compris les vaccins anti-VHA, sont commandés directement par les professionnels de la santé au Bureau de santé publique de Toronto. Par conséquent, le nombre de vaccins anti-VHA financés par la province et commandés chaque mois par les professionnels de la santé était connu et était accessible dans le module d'immunisation de Panorama, le système ontarien d'information en ligne sur l'immunisation. Un test $t$ a été effectué sur le nombre de vaccins anti-VHA commandés par mois durant l'éclosion de juin 2017 à octobre 2018, lorsque des efforts ciblés de promotion du vaccin ont été mis en œuvre dans le cadre des mesures en santé publique, par rapport au nombre de vaccins anti-VHA commandés par mois entre janvier 2012 et mai 2017, afin de déterminer si les stratégies de promotion du vaccin ont pu avoir une incidence sur les commandes de vaccins. Les vaccins utilisés par la santé publique pour les personnes d'intérêts qui ont reçu une prophylaxie post-exposition n'ont pas été inclus dans cette analyse.

\section{Mesures prises par santé publique}

Dès le début de l'éclosion, tous les enquêteurs sur les cas de VHA ont reçu une formation sur la surveillance accrue, y compris sur les facteurs de risque sexuels et l'orientation sexuelle. Ils ont également reçu une formation sur les expositions liées à la consommation de drogues illicites.

Les initiatives suivantes ont été entreprises pour promouvoir l'utilisation du vaccin en pré-exposition chez les HARSAH :

1. Des alertes ont été envoyées aux professionnels de la santé par le bulletin Toronto Public Health Communiqué, un bulletin électronique d'environ 4200 abonnés (quatre mises à jour ont été envoyées entre juin 2017 et juin 2018).

2. Trois campagnes sur les réseaux sociaux, impliquant Facebook, Instagram, Twitter et Grindr, ont été lancées pendant la période d'éclosion (novembre 2017, janvier 2018 et une en juin précédant le festival Pride Toronto 2018). Facebook et Grindr ont tous deux été sélectionnés, car ces plateformes permettent de cibler très précisément un public et parce que les utilisateurs se connectent socialement grâce à ces plateformes. Grindr, une application de réseautage géosocial orientée vers les HARSAH, a particulièrement soutenu la promotion très ciblée de vaccins auprès de cette population. Les campagnes ont été évaluées, comprenant des paramètres comme la surveillance du nombre d'impressions (combien de fois les messages étaient consultés), l'engagement (les mesures prises à la suite du message), le trafic sur le site Web du VHA du Bureau de santé publique de Toronto et l'intérêt relatif des recherches sur le Web en Ontario concernant l'hépatite A via Google Trends, une mesure d'évaluation des campagnes qui permet de mieux comprendre les tendances des recherches en ligne pendant la période de l'éclosion.

3. Un petit nombre de cliniques de vaccination anti-VHA pour les HARSAH ont été organisées dans la région de Toronto, où de nombreuses personnes lesbiennes, gaies, bisexuelles, transgenres, queer et autres (LGBTQ+) vivent et où les cas d'hépatite étaient regroupés (février 2018).

4. Des lettres ont été envoyées aux cliniques de santé sexuelle ainsi qu'aux professionnels de la santé qui traitent les infections transmises sexuellement (juin 2018).

\section{Resultats}

La figure 1 montre une courbe épidémique des cas d'éclosion par mois d'apparition des symptômes du 1er janvier 2017 au 30 novembre 2018. Au total, il y avait 42 cas confirmés et 10 cas probables. La fin de l'éclosion a été déclarée le $1^{\text {er }}$ novembre 2018 , soit deux mois après le dernier cas signalé. Au cours de l'éclosion, le Bureau de santé publique de Toronto 
a également reçu des rapports sur 46 cas de VHA qui ne correspondaient pas à la définition de cas d'éclosion.

Figure 1 : Courbe épidémique des cas confirmés et probables d'éclosion d'hépatite A à Toronto, en Ontario, par mois d'apparition des symptômes (du $1^{\mathrm{er}}$ janvier 2017 au 30 novembre 2018)

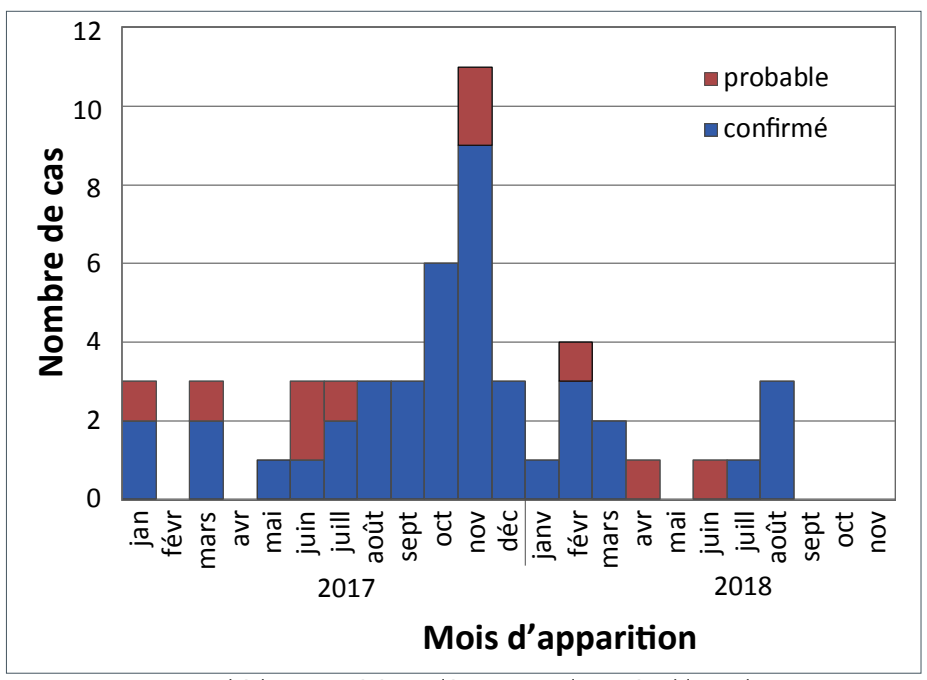

Remarque : Aucun cas d'éclosion n'a été signalé au Bureau de santé publique de Toronto en février et avril 2017 et en mai, septembre, octobre et novembre 2018

Les caractéristiques des cas confirmés et probables d'éclosion de VHA sont présentées au tableau 1. La majorité des cas d'éclosion confirmés étaient des hommes (79\%; $n=33 / 42)$, l'âge moyen étant de 38 ans. Plus de la moitié (59\%; $n=17 / 29)$ des 29 cas confirmés chez les hommes pour lesquels des renseignements étaient disponibles a déclaré un facteur de risque d'exposition à des HARSAH, par rapport à la proportion de $4 \%$ de cas de VHA ayant déclaré un facteur de risque d'exposition à des HARSAH au cours des cinq années précédentes. Près de la moitié des cas confirmés (hommes et femmes) ont déclaré avoir consommé des drogues illicites (49\%; $n=20 / 41)$ et $10 \%(n=4 / 42)$ ont déclaré être sans abri ou mal-logés. Parmi les cas confirmés, la majorité de ceux qui ont déclaré avoir consommé des drogues illicites a également déclaré un facteur de risque d'exposition à des HARSAH (65\%; $n=13 / 20)$. La consommation de drogues illicites a souvent été signalée comme un facteur de co-risque chez les HARSAH et très peu de cas ( $4 \%$ ) ont signalé une consommation de drogues illicites isolée des autres facteurs de risque. Plus de la moitié des cas confirmés ( $56 \% ; n=23 / 41$ ) a nécessité une hospitalisation. Aucun facteur de risque lié à l'éclosion n'a été signalé dans $43 \%$ ( $n=18 / 42)$ des cas confirmés.

Les résultats du séquençage ont été reçus pour 36 cas (confirmés et probables). De ce chiffre, $83 \%(n=30 / 36)$ avaient I'une des trois souches observées lors des éclosions chez les HARSAH à l'échelle mondiale. La majorité (72 \%; $n=26 / 36)$ était la souche VRD_521_2016. Cette souche d'éclosion était considérée comme la souche la plus répandue dans les
Tableau 1 : Caractéristiques des cas d'hépatite A conformes aux définitions de cas confirmées et probables à Toronto, en Ontario (du 1er janvier 2017 au 30 novembre 2018)

\begin{tabular}{|c|c|c|c|c|c|c|}
\hline \multirow{3}{*}{ Caractéristiques $^{\mathrm{a}}$} & \multicolumn{6}{|c|}{ Cas d'éclosion } \\
\hline & \multicolumn{2}{|c|}{$\begin{array}{l}\text { Confirmé } \\
(\mathrm{n}=42)\end{array}$} & \multicolumn{2}{|c|}{$\begin{array}{c}\text { Probable } \\
(\mathrm{n}=10)\end{array}$} & \multicolumn{2}{|c|}{$\begin{array}{c}\text { Total } \\
(n=52)\end{array}$} \\
\hline & $\mathbf{n}$ & $\%$ & $\mathbf{n}$ & $\%$ & $\mathbf{n}$ & $\%$ \\
\hline \multicolumn{7}{|l|}{ Sexe } \\
\hline homme & $33 / 42$ & 79 & $10 / 10$ & 100 & $43 / 52$ & 83 \\
\hline femme & $9 / 42$ & 21 & $0 / 10$ & 0 & $19 / 52$ & 17 \\
\hline \multicolumn{7}{|l|}{ Âge } \\
\hline âge moyen & 38,1 & 38,1 & 39,4 & 39,4 & 38,4 & 38,4 \\
\hline \multicolumn{7}{|l|}{ Facteurs de risque } \\
\hline HARSAH & $17 / 29$ & 59 & $8 / 10$ & 80 & $25 / 39$ & 64 \\
\hline $\begin{array}{l}\text { consommation de } \\
\text { drogues illicites }^{b}\end{array}$ & $20 / 41$ & 49 & $3 / 9$ & 33 & $23 / 50$ & 46 \\
\hline $\begin{array}{l}\text { consommation de } \\
\text { drogues illicites }{ }^{b} \text { sans } \\
\text { autres facteurs de } \\
\text { risque }\end{array}$ & $2 / 36$ & 6 & $0 / 9$ & 0 & $2 / 45$ & 4 \\
\hline $\begin{array}{l}\text { consommation de } \\
\text { cannabis }\end{array}$ & $9 / 40$ & 23 & $3 / 9$ & 33 & $12 / 49$ & 24 \\
\hline voyage & $0 / 38$ & 0 & $10 / 10$ & 100 & $10 / 48$ & 21 \\
\hline sans-abri/mal-logés & $4 / 42$ & 10 & $0 / 10$ & 0 & $4 / 52$ & 8 \\
\hline aucun signalé & $18 / 42$ & 43 & $0 / 10$ & 0 & $18 / 52$ & 35 \\
\hline \multicolumn{7}{|l|}{ Co-infections } \\
\hline co-infection par le VIH & $6 / 40$ & 15 & $3 / 10$ & 30 & $9 / 50$ & 18 \\
\hline $\begin{array}{l}\text { IST actuelles/ } \\
\text { précédentes }\end{array}$ & $13 / 40$ & 33 & $5 / 10$ & 50 & $18 / 50$ & 36 \\
\hline \multicolumn{7}{|l|}{ Morbidité } \\
\hline hospitalization & $23 / 41$ & 56 & $4 / 10$ & 40 & $24 / 51$ & 53 \\
\hline \multicolumn{7}{|l|}{ Souche } \\
\hline VRD_521_2016 & $26 / 32$ & 81 & $0 / 4$ & 0 & $26 / 36$ & 72 \\
\hline RIVM HAV16-090 & $1 / 32$ & 3 & $2 / 4$ & 50 & $3 / 36$ & 8 \\
\hline V16-25801 & $0 / 32$ & 0 & $1 / 4$ & 25 & $1 / 36$ & 3 \\
\hline non épidémique & $5 / 32$ & 16 & $1 / 4$ & 25 & $6 / 36$ & 17 \\
\hline
\end{tabular}

Abréviations : HARSAH, hommes ayant des relations sexuelles avec des hommes; IST, infections sexuellement transmissibles

a Les dénominateurs correspondent au nombre de cas où des renseignements sur chaque caractéristique ont été recueillis

b Les drogues illicites déclarées comprenaient le cannabis, le G liquide, le 3,4-méthylènedioxyméthamphétamine (MDMA, ecstasy), les opioïdes/le fentanyl, la cocaïne non injectable, la méthamphétamine en cristaux, l'héroïne et le crack

éclosions chez les HARSAH signalées dans I'Union européenne (4), ainsi que dans les éclosions en Amérique du Sud touchant les HARSAH et dans la ville de New York $(11,12)$. Une souche d'éclosion était beaucoup plus susceptible d'être séquencée parmi les cas confirmés pour lesquels au moins un facteur de risque a été signalé, par rapport à ceux pour lesquels aucun 
facteur de risque d'intérêt n'a été signalé (rapport de cotes : 8,14 ; intervalle de confiance à $95 \%: 1,32$ à 50,3 ).

Des adresses domiciliaires ont été obtenues et cartographiées pour 38 cas d'éclosion confirmés. L'indice global de Moran (test d'autocorrélation spatiale) a montré que la distribution spatiale n'était statistiquement pas aléatoire (indice de Moran : 0,15, valeur $p<0,001$ ) et on a observé un regroupement relatif dans I'un des quartiers du centre-ville de Toronto populaires auprès des communautés HARSAH et LGBTQ+.

L'évaluation des campagnes sur les réseaux sociaux a montré que les messages diffusés dans le cadre du festival Pride Toronto 2018 ont peut-être eu le plus d'impact en termes de nombre de points de vue et d'engagement envers la campagne. La figure 2 montre un graphique du terme de recherche " hépatite $A$ » dans Google pour l'Ontario au fil du temps par rapport au point d'intérêt le plus élevé, qui a coïncidé avec le festival Pride Toronto en juin 2018.

\section{Figure 2 : Nombre de recherches Google sur} "l'hépatite A » en Ontario (août 2017 à novembre 2018)

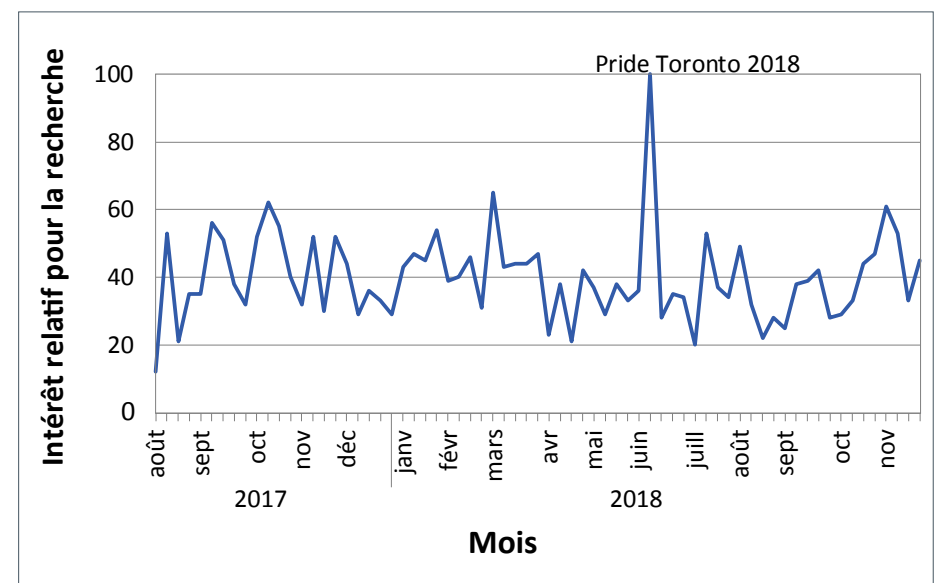

Remarques : L'axe des $\mathrm{Y}$ reflète l'intérêt de la recherche dans le temps (axe des $\mathrm{x}$ ). Le point culminant du graphique a coïncidé avec le festival Pride Toronto en juin 2018

Les commandes de vaccins anti-VHA ont augmenté au plus fort de l'éclosion (de juin 2017 à novembre 2018) par rapport à la période précédant l'éclosion. Le nombre moyen mensuel de vaccins anti-VHA commandés entre juin 2017 et octobre 2018 était de 348, contre une moyenne mensuelle de 257 de janvier 2012 à mai $2017(t=4,72, p<0,001)$. Au total, 105 vaccins ont été administrés dans les six cliniques de pré-exposition pour les HARSAH, organisées par le Bureau de santé publique de Toronto au début de 2018.

\section{Discussion}

À notre connaissance, il s'agit du premier rapport publié sur une éclosion prolongée de VHA touchant de façon disproportionnée une population de HARSAH au Canada depuis plus de vingt ans. Plusieurs éclosions des années 1990 ont été résumées (13). Puis, en 2016 et 2017, plusieurs éclosions de VHA touchant les HARSAH ont été signalées à l'échelle mondiale $(4,5,11,12)$. Durant l'éclosion de 2017-2018 déjà décrite, des regroupements ont été observés dans une région de Toronto où vivent de nombreuses personnes qui s'identifient comme faisant partie des communautés HSH et LGBTQ+. Le séquençage viral des souches de cette éclosion correspondait à celui des éclosions à l'échelle mondiale. Les réseaux sociaux et d'autres stratégies de sensibilisation employées lors d'éclosions semblables (p. ex. New York, États-Unis) ont été utilisés dans l'éclosion de Toronto pour élaborer une promotion ciblée des vaccins.

Les trois souches de VHA provenant de récentes éclosions à l'échelle mondiale touchant les HARSAH (5) ont été détectées dans cette éclosion, le VRD_521_2016 étant le plus courant. La souche VRD_521_2016 a également été la première souche à circuler lors des éclosions touchant les HARSAH dans I'Union européenne (5). D'après la courbe épidémiologique, les données sur le séquençage et les données de voyage recueillies auprès des cas signalant une exposition à des HARSAH, il est possible que les souches en circulation à l'extérieur du Canada aient été importées avant et pendant le festival Pride Toronto 2017. Cela suggère l'importation de ces souches à Toronto en juin 2017 et leur transmission locale ultérieure jusqu'à la mi-2018. Comme l'ont conclu d'autres éclosions récentes dans des zones de faible endémie, une combinaison de voyages internationaux et de réseaux sexuels peut favoriser une éclosion importante de VHA au sein d'une population vulnérable (4).

En Ontario, les HARSAH peuvent avoir accès au vaccin antiVHA pour la prophylaxie pré-exposition financé par la province. Ce programme est en place depuis 1997; toutefois, cette récente éclosion démontre que les niveaux d'immunité chez les HARSAH n'étaient pas suffisants pour éviter une éclosion. Actuellement, pour obtenir le vaccin anti-VHA financé par la province, les professionnels de la santé doivent documenter les facteurs de risque pour les HARSAH, pour la consommation de drogues ou d'autres facteurs de risque, et offrir des conseils sur les pratiques sexuelles sans risque. La nécessité de divulguer ces facteurs de risque peut réduire l'efficacité du programme de vaccination anti-VHA à risque élevé et peut constituer un obstacle au maintien d'une couverture vaccinale pré-exposition suffisamment élevée. Des études de modélisation antérieures ont estimé que des niveaux d'immunité de $70 \%$ ou plus seraient nécessaires chez les HARSAH pour prévenir une éclosion (14). La consommation de drogues illicites parmi les cas a fait l'objet d'une surveillance et il y a eu un chevauchement important entre les cas ayant signalé une exposition à des HARSAH d'une part et à la consommation de drogues de l'autre. Cette constatation peut être liée à des recherches antérieures menées auprès des HARSAH qui ont montré un lien entre l'usage de substances psychoactives et l'augmentation des comportements sexuels à risque (15). 
Les alertes aux professionnels de la santé, les réseaux sociaux et les campagnes de communication traditionnelles ont été utilisés pour accroître la sensibilisation à l'éclosion et promouvoir la vaccination. Elles ont été suivies d'une augmentation importante du nombre de commandes de vaccins anti-VHA par les professionnels de la santé de Toronto. Seulement quatre nouveaux cas ont été signalés après la campagne Pride Toronto 2018, et la fin de l'éclosion a été déclarée en novembre 2018. L'utilisation de campagnes sur les réseaux sociaux a semblé être une stratégie efficace pour sensibiliser les HARSAH à Toronto au VHA.

\section{Limites}

Cette analyse est fondée uniquement sur les cas de VHA qui ont été signalés et sous-estime probablement l'ampleur réelle de cette éclosion. Le taux d'hospitalisation observé lors de cette éclosion était plus élevé que ce qui est généralement cité pour cette maladie; $56 \%$ des cas confirmés ont nécessité une hospitalisation, ce qui, toutefois, était conforme à celui mentionné dans d'autres rapports récents d'éclosions de VHA (4). Une des hypothèses avancées est que le nombre de cas diagnostiqués est plus élevé dans les cas hospitalisés, ce qui peut entraîner une surestimation de la morbidité (4).

Pour 18 cas confirmés, il n'a pas été possible d'établir un facteur de risque d'intérêt pour la transmission de personne à personne acquise localement; leur lien épidémiologique avec l'éclosion demeure inconnu. Ces cas étaient nettement moins susceptibles d'avoir une séquence virale d'éclosion et, par conséquent, peuvent ne pas être liés à l'éclosion. Il est également possible qu'il s'agisse d'une transmission locale dans la population générale, comme cela a été signalé lors d'autres éclosions $(16,17)$. Ces cas peuvent également être mal classés, dans la mesure où ils auraient peut-être hésité à divulguer des renseignements personnels de nature délicate, notamment en ce qui concerne les activités ou l'orientation sexuelles ou la consommation de drogues illicites. À mesure de l'évolution de l'éclosion, le personnel du Bureau de santé publique de Toronto modifiait ses pratiques d'entrevue afin d'obtenir des renseignements plus précis sur les facteurs de risque pour les cas locaux de VHA. Par conséquent, les comparaisons avec les années précédentes doivent être interprétées avec prudence.

Une plateforme de commande de vaccins en ligne a été mise en place pendant la période de l'éclosion et pourrait avoir contribué à l'augmentation du nombre de commandes de vaccins. Par ailleurs, les données extraites de Panorama ne nous ont pas permis de distinguer les commandes de vaccins pour les HARSAH des autres groupes à haut risque. L'ampleur de la couverture vaccinale de base des HARSAH à Toronto est inconnue. Par conséquent, le degré de protection de cette population est également inconnu.

\section{Conclusion}

Des éclosions de VHA, causées par la transmission de personne à personne de souches de VHA qui touchent de façon disproportionnée les HARSAH, se sont maintenant produites au Canada. Ces souches ont probablement été importées d'éclosions touchant les HARSAH à l'échelle mondiale. Les populations à risque ont été identifiées à l'aide d'une combinaison de résultats de séquençage du VHA et d'une analyse descriptive de l'information sur les facteurs de risque, facilitant ainsi la promotion ciblée du vaccin. L'utilisation de campagnes ciblées sur les réseaux sociaux semble être une stratégie efficace pour promouvoir la sensibilisation au VHA et l'adoption du vaccin chez les HARSAH pendant la période d'éclosion. Une évaluation périodique des taux de couverture vaccinale chez les HARSAH et une étude plus poussée sur la meilleure façon de s'assurer de les maintenir à des niveaux élevés peuvent aider à atténuer les éclosions futures.

\section{Déclaration des auteurs}

H. S. a supervisé la gestion de l'éclosion et la rédaction du manuscrit

$M$. B. a conçu la première ébauche de ce manuscrit

S.O. et $\mathrm{M}$. B. ont analysé les données épidémiologiques

J.M. a supervisé et évalué les campagnes sur les réseaux sociaux V. D., J. M., R. S., et S. O. ont participé à la gestion de l'éclosion A. A. a fourni le séquençage et l'interprétation du virus Tous les auteurs ont participé à la rédaction et à la révision du manuscrit.

\section{Conflit d'interêts}

None.

\section{Remerciements}

Le groupe de gestion de l'éclosion d'hépatite $A$ du Bureau de santé publique de Toronto (y compris D. Hayden, K. Beckermann, $K$. Bradley et A. Summers) a facilité la collecte de données et les mesures d'intervention.

M. Finkelstein et C. C. Hui (ce dernier au nom du groupe de travail du Bureau de santé publique de Toronto sur les hommes ayant des relations sexuelles avec des hommes) ont contribué à la rédaction du manuscrit.

Le personnel et la direction de plusieurs secteurs de programme du Bureau de santé publique de Toronto ont contribué à la lutte contre l'éclosion. Les auteurs tiennent également à remercier le Laboratoire de Santé publique de l'Ontario, le Laboratoire national de microbiologie et le New York City Department of Health and Mental Hygiene pour leur soutien. 


\section{Références}

1. Agence santé publique du canada - Guide canadien d'immunisation. Partie 4 - Agents d'immunisation active : Vaccin contre l'hépatite A. ASPC; Ottawa (ON); 2018 (Accédé 2018-12-06). https://www.canada.ca/fr/ sante-publique/services/publications/vie-saine/guide-canadie n-immunisation-partie-4-agents-immunisation-active/page6-vaccin-contre-hepatite-a.html\#p4c5a2

2. Centers for Disease Control and Prevention. Chapter 9: Hepatitis A. In: Epidemiology and prevention of Vaccine Preventable Diseases (The Pink Book, 13th Edition). CDC; 2015 (Accédé 2018-12-02). www.cdc.gov/vaccines/pubs/ pinkbook/hepa.html

3. Comité consultatif national de l'immunisation (CCNI). Une déclaration d'un comité consultatif (DCC). Mise à jour des recommandations concernant I'utilisation du vaccin contre I'hépatite A. ASPC; 2016 (Accédé 2018-12-02). www.canada. $\mathrm{ca} / \mathrm{content/dam/hc-sc/documents/services/publications/}$ healthy-living/hepatitis-a-vaccine-update-recommended-use2016-fra.pdf

4. Ndumbi P, Freidl GS, Williams CJ, Mårdh O, Varela C, Avellón A, Friesema I, Vennema H, Beebeejaun K, Ngui SL, Edelstein M, Smith-Palmer A, Murphy N, Dean J, Faber M, Wenzel J, Kontio M, Müller L, Midgley SE, Sundqvist L, Ederth JL, Roque-Afonso AM, Couturier E, Klamer S, Rebolledo J, Suin V, Aberle SW, Schmid D, De Sousa R, Augusto GF, Alfonsi V, Del Manso M, Ciccaglione AR, Mellou K, Hadjichristodoulou C, Donachie A, Borg ML, Sočan M, Poljak M, Severi E; Members Of The European Hepatitis A Outbreak Investigation Team. Hepatitis A outbreak disproportionately affecting men who have sex with men (MSM) in the European Union and European Economic Area, June 2016 to May 2017. Euro Surveill 2018 Aug;23(33):1700641. DOI PubMed

5. Organisation mondiale de la santé. Flambées d'hépatite $A$ touchant principalement les hommes ayant des rapports sexuels avec des hommes - Région européenne et Région des Amériques. OMS ; 2017 (Accédé 2018-12-06). www.who.int/csr/don/07-june-2017-hepatitis-a/fr/

6. Foster M, Ramachandran S, Myatt K, Donovan D, Bohm S, Fiedler J, Barbeau B, Collins J, Thoroughman D, McDonald E, Ballard J, Eason J, Jorgensen C. Hepatitis A Virus Outbreaks Associated with Drug Use and Homelessness - California, Kentucky, Michigan, and Utah, 2017. MMWR Morb Mortal Wkly Rep 2018 Nov;67(43):1208-10. DOI PubMed

7. Ontario Ministry of Health and Long-Term Care. Publicly Funded Immunization Schedules for Ontario - December 2016. OMHLTC; 2016 (Accédé 2018-12-02). www.health.gov. on.ca/en/pro/programs/immunization/docs/immunization_ schedule.pdf

8. Ontario Ministry of Health and Long-Term Care. Infectious Disease Protocol. Appendix B: Provincial Case Definitions for Reportable Diseases. Disease: Hepatitis A. OMHLTC; 2017 (Accédé 2018-12-02). www.health.gov.on.ca/en/pro/ programs/publichealth/oph_standards/docs/hep_a_cd.pdf
9. Toronto Public Health Communicable Disease Surveillance Unit. Communicable Diseases in Toronto - 2017. www.toronto.ca/community-people/health-wellness-care/ health-inspections-monitoring/communicable-diseasestoronto/

10. Public Health Ontario. Ontario Investigation Tools (Standardized Questionnaires). PHO; 2018 (Accédé 2018-12-02). pp. 1-2. www. publichealthontario. ca/en/BrowseByTopic/InfectiousDiseases/Pages/ Standardized-Questionnaires.aspx

11. Latash J, Dorsinville $M$, Del Rosso $P$, Antwi M, Reddy $V$, Waechter H, Lawler J, Boss H, Kurpiel P, Backenson PB, Gonzalez C, Rowe S, Poissant T, Lin Y, Xia GL, Balter S. Notes from the Field: Increase in Reported Hepatitis A Infections Among Men Who Have Sex with Men - New York City, January-August 2017. MMWR Morb Mortal Wkly Rep 2017 Sep;66(37):999-1000. DOI PubMed

12. Mariojoules J, Castro G, Pisano MB, Barbero P, Fantilli A, Borda M, Canna F, Barbás G, Ré V. Hepatitis A outbreak affecting men who have sex with men (MSM) in central Argentina, occurred in July 2017-April 2018, later than the European outbreak. J Clin Virol 2019 Aug;117:49-53. DOI PubMed

13. Uhlmann S, Buxton JA. Étude provinciale et territoriale sur l'hépatite à chez les hommes ayant des relations sexuelles avec d'autres hommes. Relevé des maladies transmissibles au Canada 2007 Oct 1;33(11):1-11. https://www.canada.ca/fr/sante-publique/services/ rapports-publications/releve-maladies-transmissibles-canadarmtc/numero-mensuel/2007-33/releve-maladie s-transmissibles-canada.html

14. Regan DG, Wood JG, Benevent C, Ali H, Smith LW, Robertson PW, Ferson MJ, Fairley CK, Donovan B, Law MG. Estimating the critical immunity threshold for preventing hepatitis A outbreaks in men who have sex with men. Epidemiol Infect 2016 May;144(7):1528-37. DOI PubMed

15. Bourne A, Weatherburn P. Substance use among men who have sex with men: patterns, motivations, impacts and intervention development need. Sex Transm Infect 2017 Aug;93(5):342-6. DOl PubMed

16. Friesema IH, Sonder GJ, Petrignani MW, Meiberg AE, van Rijckevorsel GG, Ruijs WL, Vennema H. Spillover of a hepatitis A outbreak among men who have sex with men (MSM) to the general population, the Netherlands, 2017. Euro Surveill 2018 Jun;23(23):1800265. DOI PubMed

17. Lanini S, Minosse C, Vairo F, Garbuglia A, Di Bari V, Agresta A, Rezza G, Puro V, Pendenza A, Loffredo MR, Scognamiglio P, Zumla A, Panella V, Ippolito G, Capobianchi MR; Gruppo Laziale Sorveglianza Epatiti Virali (GLaSEV). A large ongoing outbreak of hepatitis A predominantly affecting young males in Lazio, Italy; August 2016 - March 2017. PLoS One 2017 Nov;12(11):e0185428-14. DOI PubMed 